\title{
Resonances in quantum-dot transport
}

\author{
Jens U. Nöckel* \\ I. Institut für Theoretische Physik \\ Universität Hamburg, Jungiusstrasse 9 \\ D-2000 Hamburg 36 \\ Federal Republic of Germany \\ Published in Physical Review B 46 (23), 15348 (1992)
}

(Received 23 June 1992)

\begin{abstract}
Scattering resonances in ballistic conduction across a quantum dot in a weak magnetic field are investigated. Due to the special geometry considered, the resonances grow narrower with decreasing $B$, until at $B=0$ they become bound states in the continuum. Whereas previously treated geometries exhibit at most one bound state with energy in the continuum, the number of such states in the present case is limited only by the number of transverse modes that the wire leads can sustain. Furthermore, the present model demonstrates the possibility of quantum-mechanical bound states in the continuum having a classical analog. The energy shifts of the resonances in a magnetic field show paramagnetic as well as diamagnetic behavior, which can be understood in terms of the dominant influence of a particular subband and its distance from the cutoff threshold in the dot region.
\end{abstract}

\section{INTRODUCTION}

Advances in nanofabrication technology have made it possible to build quantum devices that expose the wave nature of the electron in various ways. This is achieved because at low temperatures the electrons propagate coherently through the sample, the size of which is comparable to the de Broglie wavelength in one or more directions. When two degrees of freedom are quantized by the device geometry, the electronic motion is quas-ione-dimensional due to the formation of subbands. In such a structure, a two-terminal conductance experiment at vanishing bias and temperature reduces to an elastic scattering problem whose solution can be related to the conductance by the Landauer formula, [1-3]

$$
G=\frac{e^{2}}{h} \sum_{m, n}\left|T_{m n}\right|^{2}
$$

where $T_{m n}$ is the current transmission amplitude from subband $n$ to $m$, and electron spin is not considered. Being a characteristic manifestation of wave

\footnotetext{
${ }^{*}$ Current address: Department of Physics, University of Oregon. http://uoregon.edu/ $\sim$ noeckel
} 
phenomena in a scattering experiment, resonances have received considerable attention in recent nanostructure transport calculations. In particular, physical insight has been gained by establishing a connection between resonances of the open system on the one hand, and bound states on the other, typically belonging to some closely related isolated system. A well-known case in point is the interpretation by Reed et al. [4] of the fine structure in finite-bias resonant tunneling through vertical quantum dots in terms of a zero-dimensional density of states. The following list shows that this point of view has also been adopted in zero-bias calculations where Eq. (1) applies. According to Sivan, Imry, and Hartzstein[5] the Aharonov-Bohm (AB) effect in a singly connected geometry can be viewed as consecutive scattering resonances caused by the single-particle levels of the isolated dot shifting through the Fermi energy as a function of magnetic iield. A similar relationship between conductance Q oscillations and resonances due to the discrete spectrum of an isolated loop in a magnetic field is mentioned by Ravenhall, Shult, and Wyld.[6] Resonances that occur in transport through a cavity connected to wire leads have been related by Peeters[7] to the spectrum of the isolated box without leads. As an example for resonances caused by an isolated impurity in a quantum wire, Bagwell[8] shows that an attractive $\delta$-function scatterer causes exactly one quasibound state to split off below each wire subband; in analogy with a donor level in the band gap, the state splitting off from the lowest subband is truly bound, but the others lie above the continuum threshold of the wire and thus give rise to resonances. Other possibilities for resonant behavior occur clue to the finite length of the leads connected to realistic devices, when a state that would be truly bound only under the assumption of infinite leads acquires a finite lifetime. This is noted by Exner[9] for the curved quantum wire structure which has a bound state below the propagation threshold of the straight wire segments; if the leads terminate into reservoirs at a finite distance, electrons may resonantly tunnel from one contact to the other via the bound state and through the effective barriers in the wires. The multiprobe generalization[10, 11] of Eq. (1) is applied in Ref. [12] to the symmetric fourterminal cross junction. It exhibits resonances which stem from Landau orbits that get pinned at the junction at high magnetic fields (Kirczenow[13] points out that one can view the resonances as having split off from a higher-lying subband, which resembles the argument of Ref. [8]), but there is also a resonance originating from a bound state in the continuum (which we will refer to as BIC) at $B=0$; consequently, the latter resonance becomes wider with increasing magnetic field whereas the others grow narrower. Assuming that a resonance is detected in an experiment, the question is how to determine its precise physical origin. For the loop geometry[6] it is noted that the resonances are shifted so far away from the corresponding bound states of the isolated loop that a one-to-one identification is impossible. The cross junction treated in Ref. [12], on the other hand, offers a continuous and experimentally controllable parameter, namely, the magnetic field, that allows to trace the evolution of the resonances into the corresponding bound states. The same is true for the two-terminal geometry to be introduced here, which consists of an attractive dot coupled to the reservoirs by quantum wires: it exhibits bound states in the continuum at $B=0$ that become resonances when a magnetic field is turned on. The BIC can be located by elementary means because they occur in a very systematic fashion. The mechanism that causes a BIC is analogous to the quasibound states in Ref. [8]: the dot potential causes one or more bound states to split off from each wire subband; due to the special geometry considered, none of these states can couple to the scattering states that exist at the same energy when $B=0$. Only at $B \neq 0$ do they become quasibound states if they are above the continuum edge. To elaborate on this phenomenon and show its implications, the paper is organized 
as follows. The nature of the BIC is explained in Sec. II, before we make the discussion quantitative by specializing to a particular dot potential in Sec. III. The transition from BIC to resonances upon application of a magnetic field is treated in Sec. IV. Further insight into the physics of the resonant structure is gained in Sec. V by investigating the current density patterns that illustrate the electronic motion.

\section{BOUND STATES IN THE CONTINUUM}

The time-independent single-particle Schrödinger equation without magnetic field allows for square-integrable solutions that are degenerate with non-normalizable stationary states. von Neumann and Wigner proposed an explicit construction that yields such a BIC with a potential that is localized, i.e., vanishes at infinity[14]. This possibility is of particular interest in the spectroscopy of autoionizing atomic or molecular states, but also for tunneling phenomena such as nuclear decay[15, 16], and its physical origin is the destructive interference of alternative decay paths for the bound state. When one investigates nanostructures connected to infinite wire leads, new possibilities for the formation of localized orbits in the continuum of extended states arise[17]. These exotic solutions have been discovered in two of the model structures described above. Shult et al.[12] find that the symmetric cross junction exhibits exactly one bound state above the zero-point energy of the leads. Peeters[7] reports one such state for the symmetric cavity, but only for a single ratio between cavity length and wire width. In both cases, the physical reason that prevents the electron from escaping to infinity is parity conservation. The continuum is made up of the lowest subband in the wires, which has even parity while the embedded bound state is odd with respect to reflection at the wire axes. It is the infinite wire length that gives rise to a discrete energy spacing between even parity ground state and odd-parity first excited subband in the asymptotic region, between which the bound state occurs. Its odd parity effectively decouples the bound state from the continuum, but it acquires a finite lifetime when parity conservation is broken, e.g., by imperfections or a magnetic field.

Comparing the potentials that go to zero at infinity and those that do not, there is one thing they have in common despite the different mechanisms that inhibit the decay of a BIC: In all cases, the bound state is unstable under an arbitrarily small deviation of the potential from the shape or symmetry required by the respective mechanism.

This observation also applies to the continuum bound states to be discussed here, which differ from the previously cited cases in that they have a classical analog. Being of classical origin, it will be especially interesting to see these bound states evolve into quantum-mechanical scattering resonances when the instability mentioned above is induced.

Bound orbits that are degenerate with unbound ones are common in classical mechanics, a simple example being the one-dimensional double barrier potential: a particle confined between the potential barriers can have the same energy as a free particle on the outside. However, there is generally no quantum analog because barrier penetration permits the classically bound particle to escape to infinity.

Now consider the Hamiltonian

$$
H=\frac{p_{x}^{2}}{2 m}+\frac{p_{y}^{2}}{2 m}+V(x)+V^{\prime}(y),
$$

where both $V(x)$ and $V^{\prime}(y)$ are everywhere attractive, and $V(x)$ has an upper limit. Since the Hamilton-Jacobi differential equation is separable, the total en- 
ergies of the $x$ and $y$ degrees of freedom, $E^{(x)}$ and $E^{(y)}$, are both constants of the motion. The classical motion thus reduces to two independent one-dimensional problems.

Since the total energy $E$ can be distributed arbitrarily among longitudinal and transverse motion, it is possible to have $E^{(x)}$ above or below the binding threshold of $V(x)$ for one and the same value of $E$, depending only on the magnitude of $E^{(y)}$.

As is illustrated in Fig. 1, bound and free solutions coexist at that $E$ as long as $E^{(y)}$ stays in the range of bound orbits of the transverse potential $V^{\prime}(y)$. This condition can be satisfied for a large range of energies if we assume $V^{\prime}(y)$ to be much deeper than $V(x)$ (if the reverse holds, the roles of $x$ and $y$ motion simply interchange).

When the transition is made to quantum mechanics, the same conservation of $E^{(x)}$ and $E^{(y)}$ results since Eq. (2) leads to a separable Schrödinger equation. The boundstate energies are now discrete, but the classical argument above can still be applied because neither $V(x)$ nor $V^{\prime}(y)$ allow tunneling as they are purely attractive. The discreteness of the spectra for $x$ and $y$ bound orbits leads to the consequence that a BIC does not exist at arbitrary values of the total energy $E$ as for the classical analog, since $E^{(x)}$ as well as $E^{(y)}$ must coincide with an allowed energy level, as shown in Fig. 1.

Since parity conservation is not involved, this mechanism for obtaining bound states in the continuum also works with an asymmetric $V^{\prime}(y)$. In summary, we distinguish BIC that are due to destructive interference, parity-

(a)

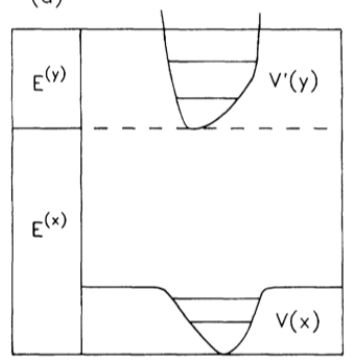

(b)

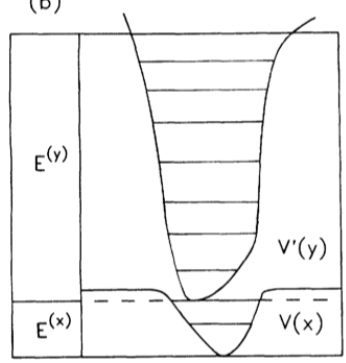

Figure 1: Example for bound classical orbit degenerate with unbound solution in two dimensions. The potentials $V(x)$ and $V^{\prime}(y)$ in the Hamiltonian (2) are everywhere attractive. In (a), the total energy $E$ is shared between longitudinal $(x)$ and transverse $(y)$ motion in such a way that $E^{(x)}$ lies in the range of free motion under the potential $V(x)$. In (b), the same total energy is redistributed, bringing $E^{(x)}$ below the binding threshold of $V(x)$ and thus resulting in a bound motion at the same value of $E$ as in (a). This classical example has a quantum analog because tunneling cannot take place. The discrete quantummechanical bound states are indicated by the solid horizontal lines. induced decoupling, and classical decoupling.

\section{QUANTUM DOT AT ZERO MAGNETIC FIELD}

The separable geometry just introduced, with a $V^{\prime}(y)$ that always yields a confined y motion, represents a special case of a quantum dot connected to infinite quantum wire leads. As is common practice in modeling narrow quantum wires, the transverse potential is taken to have the form

$$
V^{\prime}(y)=\frac{1}{2} m \omega_{0}^{2} y^{2}
$$

For the additional attractive potential $V(x)$ which defines our quantum dot, there always exists at least one bound solution, which is the only property of the $x$ potential we need in order to obtain bound states in the continuum. To 
introduce dimensionless variables, we measure frequencies in units of $\omega_{0}$, energies in $E_{0} \equiv \hbar \omega_{0}$, and lengths in $L_{0} \equiv \sqrt{\hbar / m \omega_{0}}$. If the separation of the Schrödinger equation is carried out using

$$
\Psi(x, y)=\phi(x) \chi(y)
$$

the result is

$$
\left(-\frac{1}{2} \frac{\partial^{2}}{\partial x^{2}}+V(x)\right) \phi(x)=E^{(x)} \phi(x)
$$

and

$$
\left(-\frac{1}{2} \frac{\partial^{2}}{\partial y^{2}}+\frac{1}{2} y^{2}\right) \chi(y)=\left(E-E^{(x)}\right) \chi(y)
$$

The latter is a harmonic-oscillator equation so that

$$
E^{(y)} \equiv E-E^{(x)}=n+\frac{1}{2} \quad(n=0,1 \ldots)
$$

Obviously, conservation of transverse energy $E^{(y)}$ is equivalent to the conservation of subband index $n$. To make quantitative predictions about the continuum bound states of this geometry, the quantum-dot potential $V(x)$ is now specified as a finite-depth square well of the form

$$
V(x)= \begin{cases}-V_{0} & \left(|x|>\frac{L}{2}\right. \\ 0 & \left(|x|<\frac{L}{2}\right)\end{cases}
$$

For a given $V_{0}$ and length $L$, the discrete spectrum of Eq. (5) contains a finite set of discrete eigenvalues $E_{a}^{(x)}<0(a=1,2, \ldots, N)$ that can be found by elementary methods [18]. Their number is $N=\operatorname{int}\left(\frac{V_{0} L^{2}}{\pi}\right)+1$ where $\operatorname{int}()$ denotes the integer part, which confirms that there is at least one bound state. This same set of $N$ bound solutions exists for each $n$, so that we obtain infinitely many bound states at energies $E_{n a}=E_{a}^{(x)}+n+\frac{1}{2}$. Also, if the $E_{a}^{(x)}$ are numbered in ascending order, the first eigenvalue satisfies $E_{1}^{(x)} \rightarrow 0$ as $V_{0} L^{2} \rightarrow 0$, and $E_{1}^{(x)} \rightarrow-V_{0}$ in the limit $V_{0} L^{2} \rightarrow \infty$. Therefore, the $N$ bound states that have split off from subband $n$ are constrained to lie in the interval

$$
n+\frac{1}{2}-V_{0}<E_{n a}<n+\frac{1}{2} .
$$

Since an extended state must have $E^{(x)}>0$, Eq. (7) implies that the continuum edge is $E=\frac{1}{2}$ corresponding to the $n=0$ subband threshold in the leads. In view of the discussion in Secs. IV and V, attention is restricted to the case of a single continuum in the leads, i.e., to the range of energies where only the lowest subband is populated in the wire connected to the dot. Since the propagation threshold for $n=1$ in the leads is $E=\frac{3}{2}$, the energy interval to be considered is thus

$$
\frac{1}{2}<E<\frac{3}{2} \text {. }
$$

Obviously, Eq. (9) implies that all bound states with $n=0$ are below the continuum edge. However, there will be other bound states for $n \geq 1$ that are in the energy range (10). This is illustrated by the solid lines in Fig. 2 for $V_{0}=2$. For a dot of this depth, bound states in the continuum interval (10) can be obtained with $n=1,2$, corresponding to the two distinct groups of curves in Fig. 2. The higher subbands $n \geq 3$ do not yield states in the range (10) because Eq. (9) leads to $\frac{3}{2}<E_{n a}$ in that case. The lower bound in Eq. (9) is approached asymptotically for $L \rightarrow \infty$. For the levels belonging to $n=1$, this asymptote is $E=-\frac{1}{2}$ 


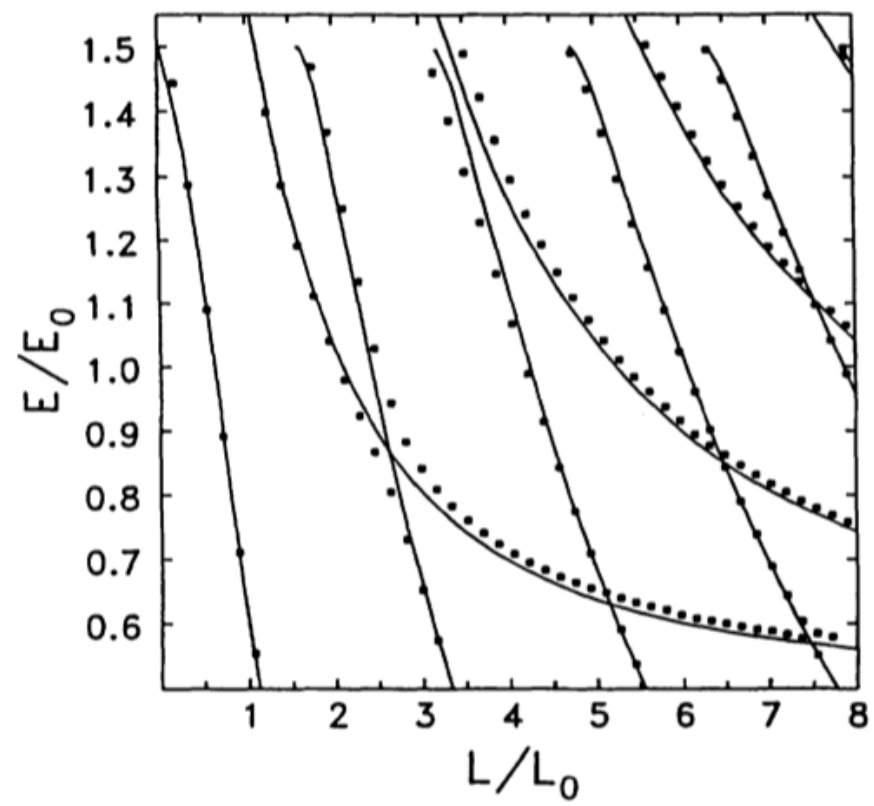

Figure 2: Solid lines: level diagram of the bound states in the continuum occurring at $\omega_{c}=0$ as a function of dot length $L$, for a fixed dot depth of $V_{0}=2 E_{0}$. Bound states with transverse quantum number $n=1$ give rise to the curves that extend below $E=0.5 E_{0}$, whereas all $n=2$ levels lie above that energy and approach it asymptotically for $L \rightarrow \infty$. Squares: points of zero transmission, indicating the presence of a resonance. A magnetic field of $\omega_{c}=0.1 \omega_{0}$ is applied to transform the bound states into metastable states.

and thus lies outside the boundary of Fig. 2, while the $L \rightarrow \infty$ asymptote of the $n=2$ curves at $E=\frac{1}{2}$ is clearly discernible in the figure. At a fixed energy, the appearance of a new bound state with index $n$ is approximately periodic in $L$ because it occurs close to a value of $L$ that permits the addition of another half wavelength to the dot. This $L$ periodicity is different for $n=1,2$ since by virtue of Eqs. (5) and (6) each subband has its own specific wavelength in the potential well. It is given at energy $E$ by

$$
\lambda_{n}(E)=\frac{2 \pi}{\sqrt{2\left(E+V_{0}-n\right)-1}},
$$

which compares well to the values of $\frac{1}{2} \lambda_{n}(E)$ obtained from the spacings in Fig. 2. In particular, $\lambda_{n}(E)$ diverges at the energy of the $L \rightarrow \infty$ asymptote, cf. Eq. (9). These observations will prove helpful in understanding the effect of a magnetic field on the position of the resonances in Sec. IV.

To complete the discussion of the $B=0$ case, the result for positive $E^{(x)}$ in Eq. (5) can be used to find the transmission probability per unit time for electrons incident from infinity in the $n$th subband:[18]

$$
\left|T_{n n}\right|^{2}=\frac{4 k^{2} \kappa^{2}}{\left|\left(k^{2}+\kappa^{2}\right) \sin \kappa L+2 i \kappa k \cos \kappa L\right|^{2}},
$$

with $k=\sqrt{2(E-n)-1}$ and $\kappa=\sqrt{2\left(E+V_{0}-n\right)-1}$.

\section{QUANTUM DOT IN A MAGNETIC FIELD}

As has been mentioned in Sec. II, one way to destabilize the parity-induced bound states in the continuum of Refs. [12] and [7] is to apply a magnetic field. This is done in Ref. [12] to the effect that a resonance appears which grows broader with increasing magnetic field $B$. The same happens to the classically decoupled bound states of the geometry considered here, because subband index 
is not conserved at $B \neq 0$. Classically, this results from the fact that the magnetic field couples $x$ and $y$ motion so that transverse energy $E^{(y)}$ is no longer a constant in the parabolic wire. If $E^{(y)}$ gets small enough just as the originally bound particle approaches a wall of the potential $V(x)$ defining the dot, it may possess sufficient longitudinal energy $E^{(x)}$ to escape to infinity. Quantum mechanically, the explanation is somewhat different because transverse energy remains a conserved quantity for the perfect quantum wire in Landau gauge. If the additional potential $V(x)$ varies rapidly on the scale of the magnetic length, this subband conservation breaks down because the assumption of adiabatic transport is then invalid.[19, 20] Since the magnetic length diverges at $B \rightarrow 0$, the abrupt rectangular well $V(x)$ of Eq. (8) becomes an increasingly realistic model for transport across a quantum dot at weak magnetic fields. This is precisely the regime of interest as far as the transition from continuum bound states to resonances is concerned. In Sec. V we will return to the question of where the resonances go at high magnetic fields.

The way in which a weak magnetic field causes subband mixing can be easily understood with the idealized potential of Eq. (8). In the Landau gauge $\mathbf{A}=$ $-B y \mathbf{e}_{\mathbf{x}}$, the Hamiltonian is

$$
H=\frac{1}{2 m}\left[\left(p_{x}-m \omega_{c} y\right)^{2}+p_{y}^{2}\right]+\frac{1}{2} m \omega_{0}^{2} y^{2}+V(x),
$$

where $\omega_{c}=\frac{e B}{m c}$ is the cyclotron frequency. The Schrödinger equation can be solved separately in the wire and dot regions where $V(x)$ is constant. There, the eigenfuntions of Eq. (13) have the product form (4) with a transverse part that is given in the reduced units of Sec. II by

$$
\chi(y)=u_{n}\left(\left[1+\omega_{c}^{2}\right]^{\frac{1}{4}}\left[y-\frac{\omega_{c}}{1+\omega_{c}^{2}} k\right]\right) .
$$

The $u_{n}$ are harmonic-oscillator wave functions, and the wave number is different in the respective $x$ intervals of Eq. (8):

$$
k=\sqrt{1+\omega_{c}^{2}} \sqrt{2[E-V(x)]-\sqrt{1+\omega_{c}^{2}}(2 n+1)} .
$$

The latter implies a different shift of the transverse wave functions in the interval $x \in\left[-\frac{L}{2}, \frac{L}{2}\right]$ and outside. This mismatch necessitates the presence of other subbands, including evanescent states, to obtain a continuously differentiable wave function at the interfaces between dot and wire leads. Consequently, the $B=0$ bound states in the continuum become metastable due to their coupling to the subbands which propagate in the leads. There are two ways to observe the finite lifetime at $B \neq 0$ : if an electron is optically excited from a bound state below the propagation threshold to one of the metastable states, the absorption spectrum will exhibit a characteristically asymmetric Fano resonance[21]. Here, we want to focus on the transport properties of the dot structure: if electrons are scattered elastically by the dot, a resonance will appear whenever the energy approaches that of a metastable state[22].

To solve the scattering problem in a magnetic field, the wave-function matching of Ref. [23] will be employed. The calculation yields the current transmission and reflection probabilities $\left|T_{m n}\right|^{2}$ and $\left|R_{m n}\right|^{2}$ between subbands $n$ and $m$, satisfying the conservation law[24]

$$
\sum_{m}\left\{\left|R_{m n}\right|^{2}+\left|T_{m n}\right|^{2}\right\}=1
$$

for all $n$. 


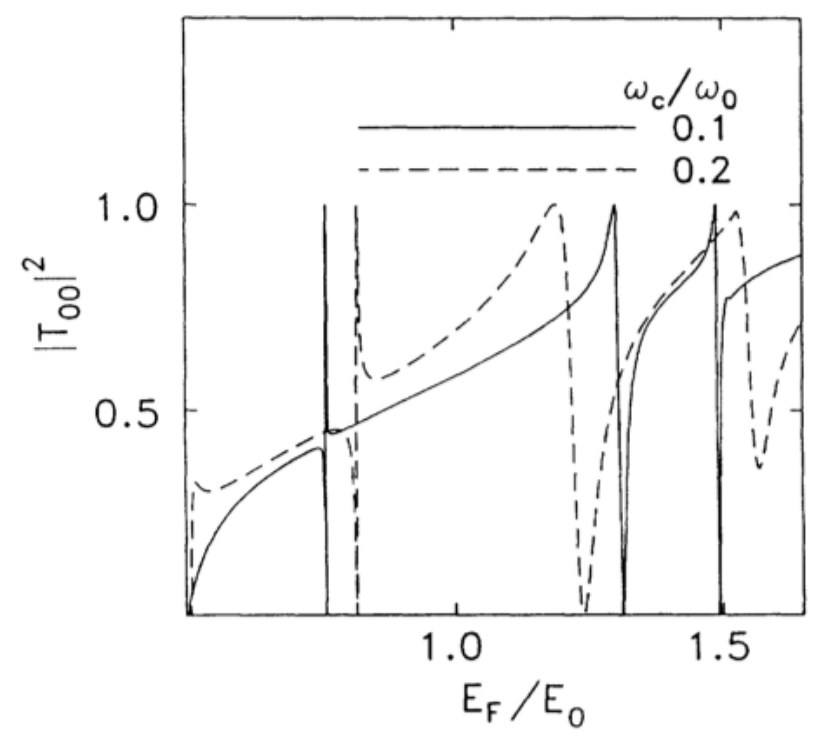

Figure 3: Current transmission as a function of energy, for dot length $L=3.5 L_{0}$ and potential $V_{0}=$ $2 E_{0}$, exhibiting asymmetric resonances that broaden and shift with increasing magnetic field. Comparison between the solid curve and Fig. 2 at $L=3.5 L_{0}$ identifies the transverse modes from which the resonances originate. The resonance which shifts to the left as $\omega_{c}$ is increased to 0.2 wo is seen to originate from an $n=1$ bound state, while the others belong to $n=2$. Additional effects arise for $E \gtrsim 1.5 E_{0}$ because a second subband is populated in the leads, cf. Fig. 4.

The resonant behavior will be most pronounced if the only propagating subband in the leads is $n=0$, because the presence of more than one populated subband can lead to cooperative effects that mask or smooth the resonant structure (see also Ref. [23]). This is the reason why we focus on the energy window (10). However, a magnetic field shifts the propagation thresholds upward and thus modifies the relevant interval to

$$
\frac{1}{2} \sqrt{1+\omega_{c}^{2}} \leq E<\frac{3}{2} \sqrt{1+\omega_{c}^{2}} .
$$

In this energy range, only the $n=0$ subband propagates in the wire, which means that only $m=n=0$ survives in Eq. (16), and the resonances will appear as rapid variations in $\left|T_{00}\right|^{2}$ as a function of $E$. In Fig. 3, the transmission at two values of the

magnetic field is shown for the parameters $V_{0}=2 E_{0}$ and $L=3.5 L_{0}$. A remarkable feature that holds for all the observed resonances as long as they are well-separated in energy is that in the neighborhood of the resonance, both $\left|T_{00}\right|^{2}=0$ and $\left|T_{00}\right|^{2}=1$ occur, leading to an asymmetric shape[25]. As expected, the resonance width decreases as $B \rightarrow 0$, since it is the magnetic field that causes the coupling between continuum and bound state. Our main task is to explain the shifts in position that these resonances obviously undergo.

But first, the effects of exceeding the upper limit of the interval (17) deserve comment. The rightmost resonance in Fig. 3 crosses the $n=1$ subband threshold when $\omega_{c}$ is raised from $0.1 \omega_{0}$ to $0.2 \omega_{0}$ wo and promptly loses its points of zero and unit transmission. In Fig. 4, a cusp in $\left|T_{00}\right|^{2}$ and an infinite slope in the conductance $G$ are resolved exactly at the onset of subband $n=1$ for the parameters of the $\omega_{c}=0.1 \omega_{0}$ curve in Fig. 3. A singularity of the same type occurs for $\omega_{c}=0.2 \omega_{0}$, where it coincides with the depressed transmission maximum. The shape of this threshold singularity has been discussed by Baranger[26]. To illustrate the one-to-one relationship between the resonances at $\omega_{c} \neq 0$ and bound states at $\omega_{c}=0$, the resonance energies at a small but nonzero magnetic field of $\omega_{c}=0.1 \omega_{0}$ have been superimposed on the bound-state curves in Fig. 2. Here, we have identified the resonance energy with the position of $\left|T_{00}\right|^{2}=0$, which is 


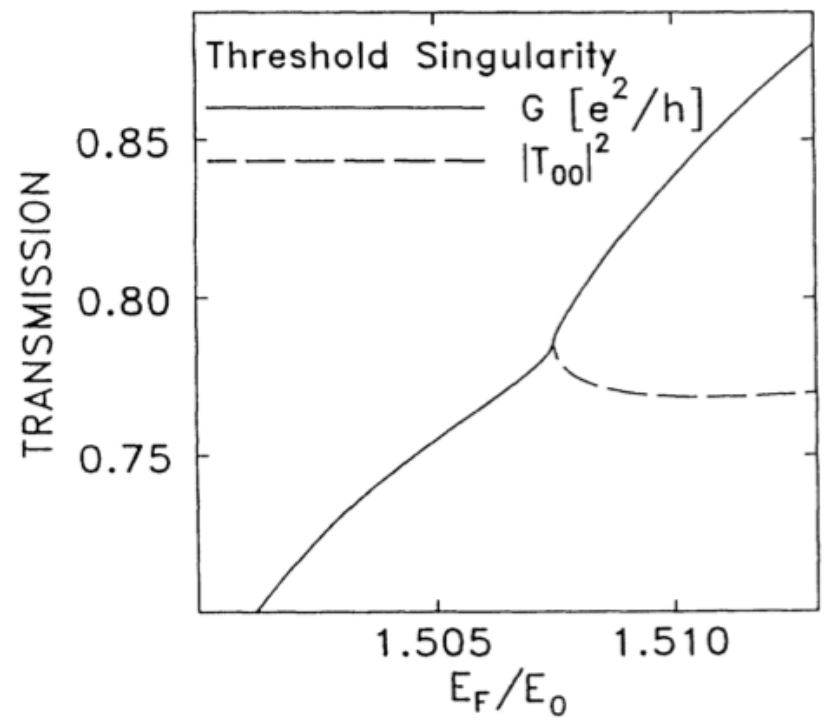

Figure 4: Enlarged plot of the threshold singularity occurring in Fig. 3 for $\omega_{c}=0.1 \omega_{0}$ at $E_{F}=$ $1.507 E_{0}$. Not shown is the similar behavior for $\omega_{c}=0.2 \omega_{0}$ at $E_{F}=1.530 E_{0}$. As in Fig. 3 , the parameters are $L=3.5 L_{0}$ and $V_{0}=2 E_{0}$. The curves for $\left|T_{00}\right|^{2}$ and the dimensionless conductance $G$ in units of $\frac{e^{2}}{h}$ coincide to the left of the singularity.

accurate for the rather sharp resonances at small $\omega_{c}$. Still, there are systematic deviations between resonance and bound-state curves in Fig. 2 which are due to the fact that the resonances do not only change in width as we is varied, but also in position.

The resonances in Fig. 3 are characteristic of the different types of magneticfield dependence seen in Fig. 2: resonances originating from $n=2$ bound states are always shifted to higher energies when $\omega_{c}$ increases, i.e., they behave diamagnetically. Resonances belonging to

$n=1$ show diamagnetic behavior near the bottom of the energy window, but have a paramagnetic character at higher energies. We will now give an explanation for these different tendencies.

Consider first the diamagnetic resonances originating from the $n=2$ bound states. In spite of the admixture of different subbands by the magnetic field, the dominant contribution to the metastable states will continue to be $n=2$. The effect of $\omega_{c} \neq 0$ is to raise the $n=2$ subband bottom in the dot above the original value of $E=\frac{1}{2}$, lifting with it the $L \rightarrow \infty$ asymptote in Fig. 2, and thus raising the $n=2$ curves. This can be seen clearly in the divergence of the subband-specific wavelength, which is given for $\omega_{c} \neq 0$ by

$$
\lambda_{n}(E)=\frac{2 \pi}{\sqrt{1+\omega_{c}^{2}} \sqrt{2\left(E+V_{0}\right)-\sqrt{1+\omega_{c}^{2}}(2 n+1)}}
$$

instead of Eq. (11). Obviously, the wavelength of the dominant subband $n$ in a metastable state diverges at $E=\sqrt{1+\omega_{c}^{2}}\left(n+\frac{1}{2}\right)-V_{0}$; hence the diamagnetic shifts of the $n=2$ resonances. We cannot predict the exact resonance energies with this simple argument because anticrossings between the $n=1$ and 2 resonances lead to additional complications. In Fig. 2, a conspicuous example for an avoided crossing can be seen for the first $n=2$ level and the second $n=1$ level. Other anticrossings are not as clear because our method of identifying the resonance position with $\left|T_{00}\right|^{2}=0$ fails when two of them occur close together. In that case, energies of zero and unit transmission no longer occur in pairs that can be attributed uniquely to one resonance.

The resonances belonging to $n=1$ show paramagnetic behavior toward the top of the energy window of Fig. 2, i.e., they shift in the direction opposite to that 


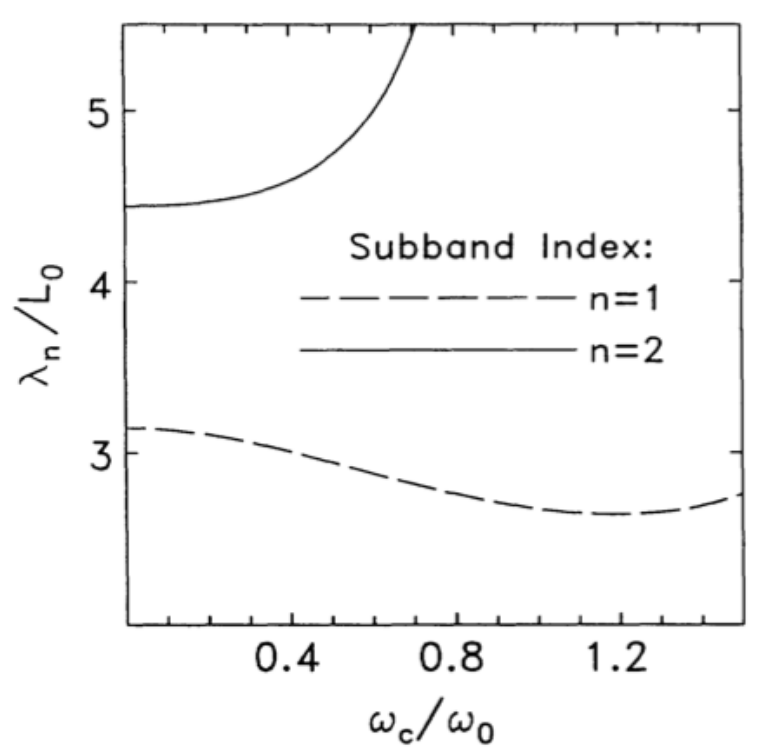

Figure 5: Subband-specific wavelength $\lambda_{n}$. from Eq. (18), plotted as a function of magnetic field at $E_{F}+V_{0}=3.5 E_{0}$ which corresponds to $E_{F}=1.5 E_{0}$ in Fig. 2. To raise the $n=1$ subband bottom to this energy and thus force $\lambda_{1}$ to diverge, a magnetic field of $\omega_{c} \approx 2.1 \omega_{0}$ is required.

of the $n=2$ resonances. The exact shifts are influenced by avoided crossings, but the paramagnetic tendency can again be understood under the assumption that the metastable state consists mainly of the original $n=1$ bound state. To show this, $\lambda_{n}$ from Eq. (18) is plotted in Fig. 5 as a function of magnetic field for $V_{0}=2$ and $E=\frac{3}{2}$, i.e., $E+V_{0}=3.5$. If we choose a fixed energy near $E=\frac{3}{2}$ and increase the dot length $L$, we can expect the average spacing between new resonances to be roughly $\frac{1}{2} \lambda_{1}$, in analogy with the bound states of Sec. II. Since $\lambda_{1}$ decreases with $\omega_{c}$, resonances shift to lower $L$ at fixed $E$. The correct overall behavior in Fig. 2 is thereby obtained. In the same way, the fact that $\lambda_{2}$ grows with $\omega_{c}$ because of the proximity to the subband bottom in the dot region is found to be in accordance with the diamagnetic behavior of the $n=2$ resonances discussed above. Unlike for $n=2$, the bottom of the $n=1$ subband in the dot is still far enough below $E$ to allow a decrease in wavelength $\lambda_{1}$ as we is increased from zero. The possibility of a decreasing $\lambda_{n}$ as a function of $\omega_{c}$, or equivalently an increasing wave number $k$, can be identified as purely quantum mechanical by noting that the group velocity always decreases monotonically with growing $\omega_{c}$. The physical reason for the increasing $k$ at fixed $E$ is a flattening of the dispersion relation $E$ vs. $k$ as its parabolic branches evolve into degenerate Landau levels with growing $\omega_{c}$. When $E$ is close to subband cutoff in the dot, as for $n=2$ in Fig. 5, this effect is outweighed by the simultaneous depopulation of the respective level.

\section{CURRENT DENSITY PATTERNS}

In the preceding section, we have succeeded in understanding how the magneticfield dependence of the resonances follows from the influence of the "parent" subband. But other subbands, including evanescent states, are also present in the metastable state. In fact, they are indispensable for obtaining the coupling between the $n=0$ subband in the wire and the transverse wave function of the continuum bound state which has $n \geq 1$. The electronic motion is therefore no longer genuinely one dimensional as in the cases $B=0$ or $V_{0}=0$. This dimensionality crossover is evident in the current density. For one-dimensional 
propagation, the wave function has the product form of Eq. (4) which leads to a current density $\mathbf{j}=j(x, y) \mathbf{e}_{\mathbf{x}}$ because $\chi$ is real. Flux conservation then requires $j(x, y)$ to be independent of $x$, i.e.,

$$
\mathbf{j}=j(y) \mathbf{e}_{\mathbf{x}} .
$$

When subband mixing is present, it is clear that such a simple expression cannot hold since interference terms and evanescent states give rise to nonzero $j_{\mathbf{y}}$. A special case exists at a resonance minimum where $\left|T_{00}\right|^{2}=0$. The probability flux must vanish at any cross section through the wire because $n=0$ is the only subband propagating to infinity. To the right of the dot, the wave function has the asymptotic form (4) where the transverse part is now the shifted harmonicoscillator ground-state function from Eq. (14),

$$
\chi_{k 0}(y)=u_{0}\left(\left[1+\omega_{c}^{2}\right]^{\frac{1}{4}}\left[y-\frac{\omega_{c}}{1+\omega_{c}^{2}} k\right]\right) .
$$

This implies that $\mathbf{j}$ must vanish identically for $x \rightarrow \infty$. To the left, the asymptotic wave function contains a reflected wave whose transverse part is shifted by the same amount as that of the incident wave, but in the opposite direction:

$$
\Psi(x, y) \propto e^{i k x} \chi_{k 0}(y)+e^{-i(k x+\delta)} \chi_{-k 0}(y) .
$$

The transverse shift being due to the Lorentz force, we can achieve $\chi_{k 0}(y)-$ $\chi_{-k 0}(y) \rightarrow 0$ by choosing $\omega_{c}$ sufficiently small. In that limit, $\Psi(x, y)$ in Eq. $(21)$ attains the product form (4) and $\mathbf{j}$ must hence vanish for $x \rightarrow-\infty$. We are then left with a current density that vanishes everywhere except for the vicinity of the dot. This reflects the motion of an electron captured in the metastable state for the limiting case of small coupling to the continuum. The current density patterns shown in Fig. 6 were calculated at $\omega_{c}=0.1 \omega_{0}$ close to the energies of zero transmission in Fig. 3. These plots shed new light on the magnetic-field dependence of the resonances which we termed paramagnetic and diamagnetic in Sec. IV: the metastable electronic orbits take on the shape of vortices with a dominant sense of rotation that corresponds to a positive or negative magnetic dipole moment. Comparison with Fig. 3 shows that diamagnetic behavior is matched by a counterclockwise rotation, while paramagnetism comes with clockwise vorticity. This can be called a normal Zeeman effect of the metastable state.

At first sight, one might expect that the area covered by individual vortices is determined by the requirement of an integer multiple of flux quanta per vortex, as in Landau diamagnetism. But we are dealing with the limit $\omega_{c} \rightarrow 0$, where the cyclotron radius is much larger than the dot dimensions. Consequently, the electronic motion is dominated by the dot geometry, which means the vortices are simply the result of interferences between the subbands that make up the quasibound state. The primary effect of the magnetic field is to trigger vortex formation by causing the subband mixing in the first place. Flux quantization is not obeyed by the vortices because they, like the whole system, are not completely closed as long as we $\omega_{c} \neq 0$.

It seems contradictory that the current density pattern of a resonance should simply be due to geometry-related interferences and thus be constant in shape for small $\omega_{c}$, whereas none of the vortices can exist at all in the absence of a magnetic field. This unphysical singularity of the current density pattern for $\omega_{c} \rightarrow 0$ results from the failure of the independent electron approximation when the resonance width goes to zero. As the lifetime of the metastable state diverges, so does the number of electrons in the dot region, because in the absence of interparticle scattering each injected electron stays in the quasibound orbit for an 
arbitrarily long time. An increase of the charge in a metastable state is predicted in Ref. [27] for the rectangular cavity at $B=0$, but the importance of the effect is described as secondary. The situation is different here because only the inclusion of electron-electron scattering will render the dimensionality crossover continuous. Both the positions and shapes of the resonances will thus start to deviate from our predictions when the independent-particle model, on which all the literature cited in the Introduction is based, breaks down in the vicinity of $\omega_{c}=0$. To create $V^{\prime}(y)$ and $V(x)$, respectively, we suggest the combined use of in-plane and surface gating[28]. Exact separability of the Schrödinger equation at $B=0$, just like the conservation of parity required for the BIC in Refs. [7] and [12], will be impossible to realize experimentally, due to inaccurate alignment of the gates and the presence of disorder[29]. These deviations from separability were not considered here because the magnetic field serves the same purpose, namely, to destabilize the BIC and thus create resonances. If, therefore, the resonances retain a finite width at $B=0$, the $B \rightarrow 0$ singularity in current density and charge does not occur.

Summarizing the independent-particle results of this and the preceding section, we obtain the following picture: even at arbitrarily small magnetic fields, the electrons captured in the metastable state move in vortices that have a net magnetic moment. Since a resonance originating from subband $n$ behaves diamagnetically or paramagnetically depending on the energetic distance to the bottom of subband $n$ in the dot region, it can be concluded that the sense of rotation is clockwise when subband $n$ is still far from being cut off in the dot at the resonance energy, and counterclockwise if that is not the case.

This relationship is not obvious from analogies with transport at higher magnetic fields. It must be kept in mind that the vortices form only near the resonances, whereas the current density is more or less of the laminar form (19) otherwise. This distinguishes our resonances from the AB effect in a singly connected geometry as reported in Ref. [30]. There, the formation of edge channels at high magnetic fields is essential. Our paramagnetic resonances have a current density profile reminiscent of such edge channels, but they are not involved in the high-field $\mathrm{AB}$ effect. This can be seen by following the upward shift of the subband thresholds in the dot as $\omega_{c}$ grows. Generalizing Eq. (9) to we $\omega_{c} \neq 0$, we note that resonances splitting off from subbands $n \geq 1$ cannot occur below the energy $E_{1}=\frac{3}{2} \sqrt{1+\omega_{c}^{2}}-V_{0}$, which is the $n=1$ subband bottom in the dot region. This approaches the $n=1$ subband bottom of the wire leads in the limit $\omega_{c} \rightarrow \infty$. As a result, the resonances are pushed out of the energy window (17) and thus cannot affect transmission in the single-subband regime. This is consistent with the high-field formula

$$
\left|T_{00}\right|^{2}=\frac{T_{A} T_{B}}{1+R_{A} R_{B}-2 \sqrt{R_{A} R_{B}} \cos \frac{e \Phi}{\hbar c}},
$$

where $R_{A / B}, T_{A / B}$ are phenomenologically or experimentally determined reflection and transmission probabilities of left and right interfaces between dot and wire leads, and $\Phi$ is the magnetic flux enclosed by the one-dimensional edge channels encircling the dot. The AB oscillations described by Eq. (22) as a function of $\Phi$ do not go through zero because the resonant structure due to interferences within the $n=0$ level itself is weaker than that caused by the BIC. Another expression for the high-Held limit of $\left|T_{00}\right|^{2}$ has been given in Ref. [31] for our model potential, but it is of very limited validity.[32] Still it agrees with the above argument because it is precisely of the one-dimensional form (12), with $k$ and $\kappa$ given by Eq. (15), which means that $\left|T_{00}\right|^{2}$ has no zeros above the continuum threshold. 
(b)

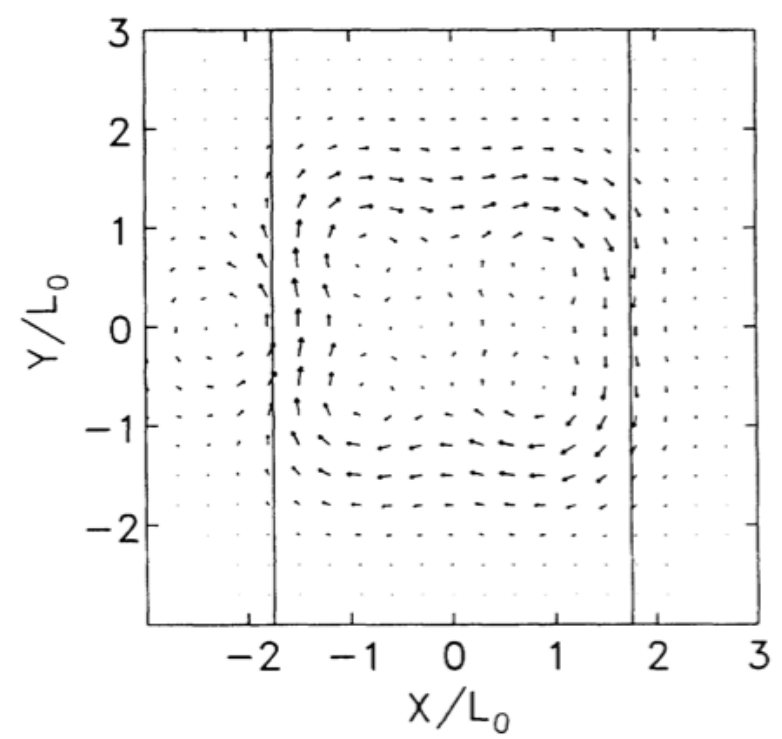

(c)

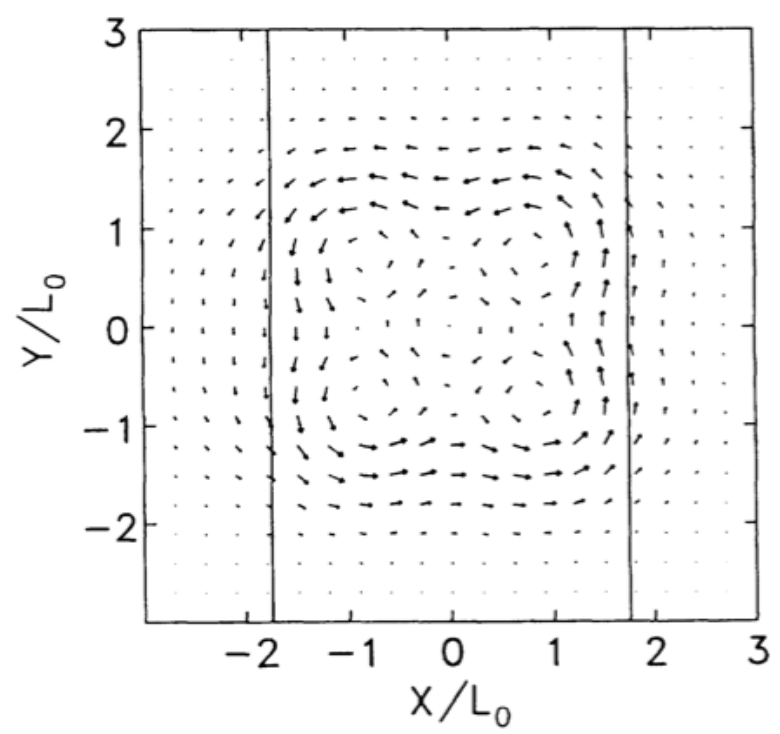

Figure 6: Current density profiles at $\omega_{c}=0.1 \omega_{0}$ for the three transmission minima of Fig. 3 in order of increasing energy. The boundaries of the dot region of length $L=3.5 L_{0}$ are indicated by solid vertical lines. The current density rotates clockwise for the paramagnetic metastable state of (b), whereas (a) and (c) show counterclockwise vorticity in agreement with their diamagnetic energy shifts observed in Fig. 3. 


\section{CONCLUSION}

We have investigated transport in a model geometry consisting of straight wire and dot potential, which is separable in the absence of a magnetic field and exhibits bound states in the continuum that split off from each wire subband. Their existence can be understood classically, which is due to the fact that quantummechanical tunneling does not make the classically bound states metastable, as is usually the case. Upon application of a weak magnetic field, sharp and asymmetric resonances develop out of the bound states. Their energy shift can be upward or downward with increasing magnetic field, depending on the change in wavelength of the "parent" subband from which they split off. The different magnetic-field dependences are clearly reflected in the current densities associated with the respective metastable states. This allows us to conclude that the electronic orbit of a metastable state possesses a paramagnetic dipole moment when the "parent" subband is far from cutoff, whereas the quasibound state has a diamagnetic vorticity if it has split off from a subband close to threshold. The highly systematic behavior of the resonances, which is insensitive to deviations of $V(x)$ or $V^{\prime}(y)$ from the shape assumed in this paper, leads to the expectation that the present geometry is suitable for an experimental study.

The author thanks R.R. Gerhards for stimulating this work, and H. Heyszenau for accompanying its progress with helpful discussions.

\section{References}

[1] R. Landauer, Z. Phys. 68, 217 (1987).

[2] D.S. Fisher and P.A. Lee, Phys. Rev. B 23, 6851 (1981).

[3] A.D. Stone and A. Szafer, IBM J. Res. Dev. 32, 384 (1988).

[4] M.A. Reed et al., Phys. Rev. Lett. 60, 535 (1988).

[5] U. Sivan, Y. Imry, and C. Hartzstein, Phys. Rev. B 39, 1242 (1989)

[6] D.G. Ravenhall, R.L. Shult, and H.W. Wyld, Superlatt. Microstruct. 11, 69 (1992).

[7] F.M. Peeters, in Science and Engineering of One- and ZeroDimensional Semiconductors, edited by S.P. Beaumont and C.M. Sotomajor Torres (Plenum, New York, 1990).

[8] P.F. Bagwell, Phys. Rev. B 41, 10354 (1990).

[9] P. Exner, Phys. Lett. A 141, 213 (1989).

[10] M. Büttiker, Phys. Rev. Lett. 57, 1761 (1987).

[11] M. Büttiker, IBM J. Res. Dev. 82, 317 (1988).

[12] R.L. Shult et al., Phys. Rev. B 41, 12760 (1990).

[13] G. Kirczenow, Phys. Rev. Lett. 82, 2993 (1989).

[14] J. von Neumann and E. Wigner, Phys. Z. 30, 465 (1929).

[15] F.H. Stillinger and D.R. Herrick, Phys. Rev. A 11, 446 (1975).

[16] H. Friedrich and D. Wintgen, Phys. Rev. A 32, 3231 (1985). 
[17] By assuming the confining potentials to extend out to infinity, resonances of the type suggested by Exner (Ref. [9]) are ruled out. This is not a severe shortcoming if we make the real leads sufficiently long to reduce any resonant tunneling widths below experimental resolution, which in turn cannot be better than of the order $k_{B} T$ due to the rounding of the Fermi function; S. Datta, Superlatt. Microstruct. 6, 83 (1989).

[18] L.I. Schiff, Quantum Mechanics (McGraw-Hill, Singapore, 1968).

[19] L.I. Glazman and M. Jonson, J. Phys. Condens. Matter 1, 5547 (1989).

[20] M. Büttiker, Phys. Rev. B 38, 9375 (1988).

[21] U. Fano, Phys. Rev. 124, 1866 (1961).

[22] The coupling between continuum and bound states in our model is different from the Feshbach coupling assumed in Ref. [16] in that our interaction is caused solely by the magnetic field and therefore cannot be described by real coupling potentials between open and closed channels, as is done when dealing with configuration interaction. The prediction of Ref. [16] that interfering resonances bring about new bound states in the continuum therefore does not apply here.

[23] J.U. Nöckel, Phys. Rev. B 45, 14225 (1992).

[24] P. Streda, J. Kucera, and A.H. MacDonald, Phys. Rev. Lett. 59, 1973 (1987).

[25] This is a further indication that the Feshbach theory leading to Breit-Wigner resonances in Ref. [16] cannot be used here.

[26] H.U. Baranger, Phys. Rev. B 42, 11479 (1990). The treatment in Appendix B contains an error in Eq. (B7) where the logarithmic derivative cannot, in general, be set equal to a constant. The resulting Eqs. (B8) and (B9) are thus incorrect except for special cases and must be replaced by the more generally applicable Eqs. (B12) and (B15) whose validity is confirmed in the present study.

[27] C.S. Lent, Appl. Phys. Lett. 57, 1678 (1990). The current density plots in this reference confirm once more that vortices are a simple consequence of interferences and as such not surprising.

[28] A.D. Wieck and K. Ploog, Appl. Phys. Lett. 56, 928 (1990).

[29] J.A. Nixon and J.H. Davies, Phys. Rev. B 41, 7929 (1990).

[30] B.J. van Wees et al., Phys. Rev. Lett. 62, 2523 (1989).

[31] J.K. Jain and S.A. Kivelson, Phys. Rev. B 37, 4276 (1988).

[32] In particular, its use in P.H.M. van Loosdrecht et al., Phys. Rev. B 38, 10162 (1988) is unwarranted. To see this, note that the limiting process of Ref. [31] leaves all lengths constant in units of the magnetic length while taking $\omega_{c} \rightarrow \infty$. This means that the resulting expression for the transmission coefficient in field-independent units is valid only if the edge channel displacement and the scattering potential are vanishingly small. For an abrupt potential like 
the one under consideration, the method of restricting the motion to the lowest Landau level [S.M. Girvin and T. Jach, Phys. Rev. B 29, 5617 (1984)] is not applicable because the potential always stays abrupt on the scale of the magnetic length. Physically, this is not a failure of the method but of the assumed model potential at high magnetic fields. 